\title{
Velocity field measurements in an evaporating sessile droplet by means of micro-PIV technique
}

\author{
Anna Yagodnitsyna ${ }^{1,2, a}$, Artur Bilsky ${ }^{1,2}$, Mina Roudgar ${ }^{3}$, Joël De Coninck ${ }^{3}$ and Oleg Kabov ${ }^{1,4}$ \\ ${ }^{1}$ Kutateladze Institute of Thermophysics SB RAS, Novosibirsk, Russia \\ ${ }^{2}$ Novosibirsk State University, Novosibirsk, Russia \\ ${ }^{3}$ Laboratory of Surface and Interfacial Physics. Université de Mons, Belgium \\ ${ }^{4}$ National Research Tomsk Polytechnic University, Tomsk, Russia
}

\begin{abstract}
Velocity fields are measured in evaporating sessile droplets on two substrates with different contact angles and contact angle hysteresis using micro resolution particle image velocimetry technique. Different flow patterns are observed in different stages of droplet evaporation: a flow with vortices and a radial flow. Flow structure is found to be similar for droplets on different substrates.
\end{abstract}

\section{Introduction}

The interest towards droplet evaporation is connected with number of scientific and technological processes such as DNA molecules visualization, ink-jet printers, spray cooling and so on [1-3]. Flow structure in evaporating droplets can dramatically influence the heat and mass transfer processes and particle deposition on a substrate. Hence, experimental data of velocity fields inside evaporating droplets are highly demanded not only for technological processes design and optimization but also for numerical code verification. A number of works have been focused on experimental study of flow structure in evaporating sessile droplets. The authors of the work [4] used the ray tracing method in order to correct optical disturbances during PIV measurements from the side view of the droplet. In the work [5] micro-PIV technique was used for velocity field measurements in the small region near the contact line of evaporating water droplet on a glass substrate. In the present work in order to visualize flow structure in evaporating droplets on the substrates with different contact angles microPIV technique is applied.

\section{Experiment details}

The scheme of experimental setup is shown in Figure 1. Micro-PIV system is used for velocity field measurements in an evaporating sessile droplet. The system is made of Zeiss Axio Observer.Z1 microscope with 10x/0.25 lens and motorized stage, double-pulsed Nd:YAG green laser, double exposure CCD camera with 11 Mpix resolution and a personal computer with ActualFlow software for data acquisition, processing and visualization. The motorized stage is able to move in three directions with 1 micrometer resolution. It is used for accuretly moving the focal plane. The working

\footnotetext{
${ }^{a}$ Corresponding author : yagodnitsinaaa@gmail.com
} 
fluid is an ultra-pure water seeded with $3.2 \mu \mathrm{m}$ fluorescent polystyrene particles. Droplets are created by high precision micropipette. The droplet volume is equal to $1.5 \mu \mathrm{l}$ with $1 \%$ accuracy. During experiments the environment temperature and the humidity has been controlled. Evaporation time for each droplet has been also measured as a time between droplet pipetting and its dry-out The standard deviation of evaporation time is not exceed $12 \%$ for each substrate.

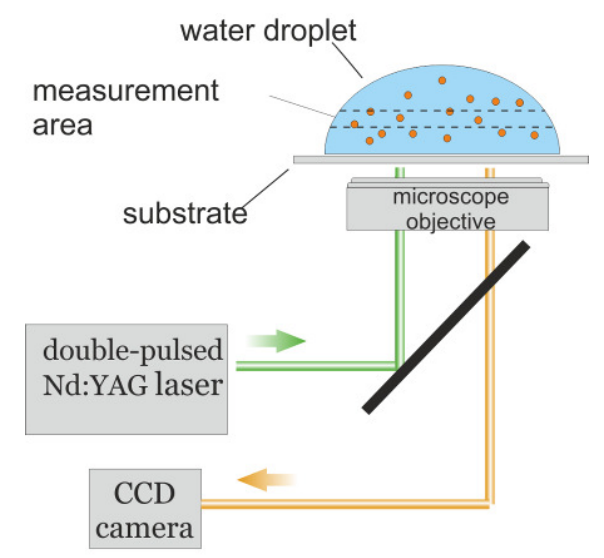

Figure 1. The scheme of experimental setup.

Experiments have been performed for two transparent substrates with different receding contact angles (RCA) and contact angle hysteresis (CAH): the substrate covered by octadecyltrichlorosilane monolayer (OTS) and the substrate covered by SU-8 photoresist. The contact angle and contact angle hysteresis measured on DSA 100 (Kruss) are presented in the Table 1.

Table 1. The properties of the used substrates.

\begin{tabular}{|c|c|c|}
\hline Substrate & RCA & CAH \\
\hline OTS & $95^{\circ} \pm 5^{\circ}$ & $10-15^{\circ}$ \\
\hline SU-8 & $65^{\circ} \pm 5^{\circ}$ & $30^{\circ}-35^{\circ}$ \\
\hline
\end{tabular}

Droplets are illuminated and observed from the flat bottom side, so optical distortions are not contribute to the measurement results. Velocity field measurements are performed in 9 cross-sections parallel to the substrate plane located at different distances from the substrate ranging from $13 \mu \mathrm{m}$ to $465 \mu \mathrm{m}$. The depth of correlation is equal to $55 \mu \mathrm{m}$. For each cross-section several measurements for different droplets are done in order to obtain sufficient statistical information. The measurements are carried from the beginning of droplet evaporation up to the end.

In-house software package "ActualFlow" is used to process the acquired data. Image preprocessing is included masking and minimum intensity subtraction calculated for every 20 particle images. These procedures allow eliminating background noise and decreasing out-of-focus particles influence. Instantaneous velocity fields are calculated using iterative cross-correlation algorithm. Final spatial resolution of measured instantaneous velocity fields is equal to $30 \mu \mathrm{m}$ per velocity vector. 


\section{Results}

Particle trajectories visualization and instantaneous velocity fields at different time starting from the beginning of droplet evaporation at the distance $93 \mu \mathrm{m}$ from the SU-8 substrate plane are presented in Fig. 2 and Fig. 3. One can see that at the beginning of evaporation flow structure does not present typical radial flow [5]. Instead, there are two symmetrical vortices in the droplet, which presumably are connected with some local depinning of the contact line. The maximum velocity near the edges are not exceed $6 \mu \mathrm{m} / \mathrm{s}$. When the height of the droplet becomes smaller the flow turns into radial with higher velocities near the edges of the droplet. In this evaporation stage maximum velocity decreases significantly down to $1.5 \mu \mathrm{m} / \mathrm{s}$. The similar flow in an evaporating droplet is found in threedimensional direct numerical simulations in [6]. Analysis of velocity fields in evaporating droplet on the OTS substrate shows the same behaviour of the flow as in evaporating droplet on SU-8 one. During the time between 78 and 220 seconds from the beginning of evaporation for OTS substrate the droplet cross-section radius has been decreased by approximately $30 \%$. In comparison for SU-8 substrate the droplet cross-section radius reduction is equal to $15 \%$ which is consistent with the statement that the large-contact-angle hysteresis droplets can be pinned [7].
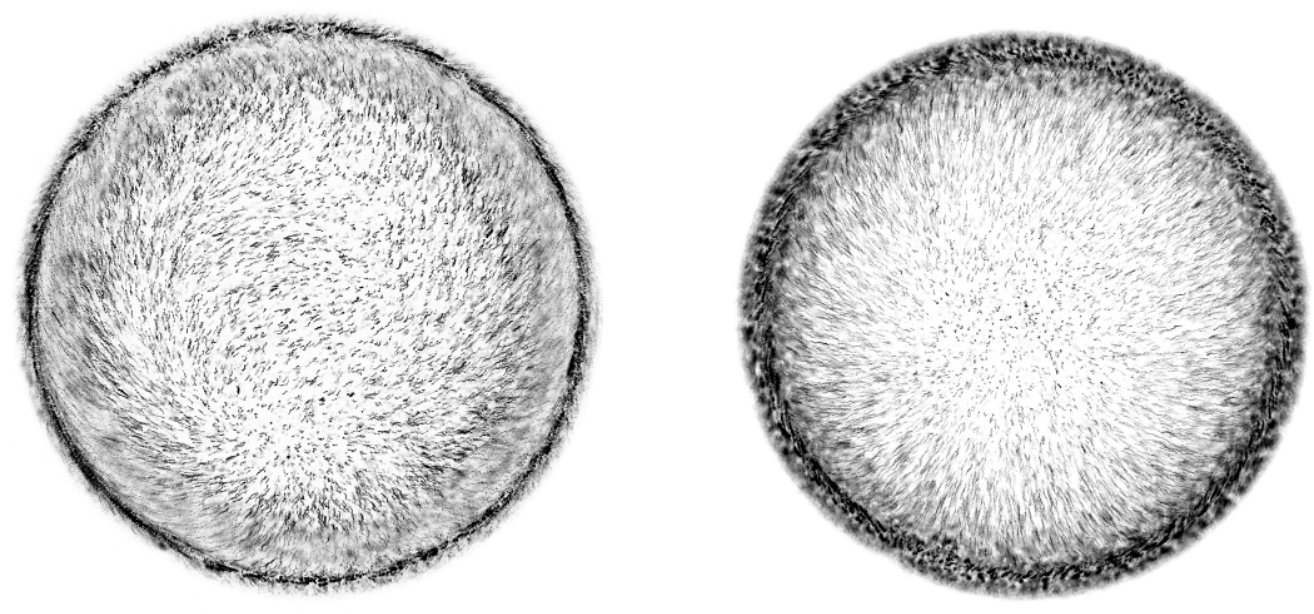

Figure 2. Trajectories of seeding particles in the droplet at the distance $93 \mu \mathrm{m}$ from the SU-8 substrate. Left: $78 \mathrm{~s}$ past the beginning of evaporation. Right: $220 \mathrm{~s}$ past the beginning of evaporation.

\section{Conclusion}

In order to visualize flow structure in evaporating sessile droplets on substrates with different properties micro-PIV technique has been used. Instantaneous velocity fields are measured at different distances from substrate plane. Analysis of instantaneous velocity fields shows that flow structure in evaporating droplet changed significantly during the evaporation process. At the first stage of evaporation, two vortices have been found in the droplet. Similar vortices have been obtained for droplets on both substrates. At the second stage of evaporation droplet height decreases and velocity fields become radial with higher velocities near the edges of the droplets.

\section{Acknowledgments}

This work was supported by a grant from the Russian Science Foundation (project 14-19-01755). 

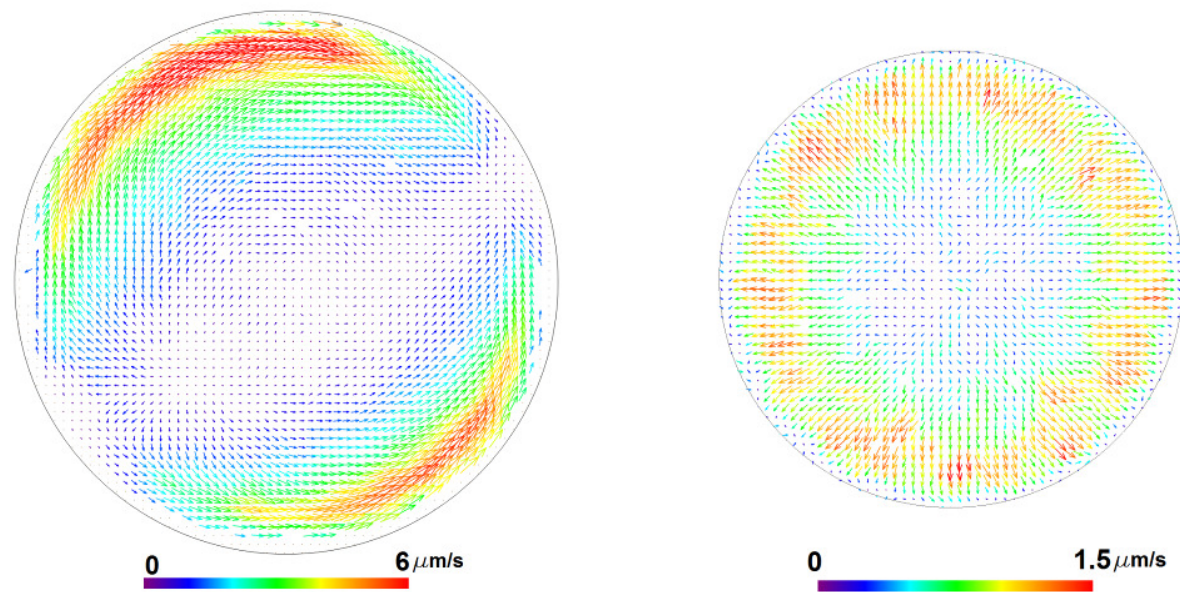

Figure 3. Instantaneous velocity fields in a droplet cross-section located at the distance $93 \mu \mathrm{m}$ from the substrate surface made of SU-8. Left: $78 \mathrm{~s}$ after the beginning of evaporation. Right: $220 \mathrm{~s}$ after the beginning of evaporation. Full evaporation time is equal to $558 \mathrm{~s}$.
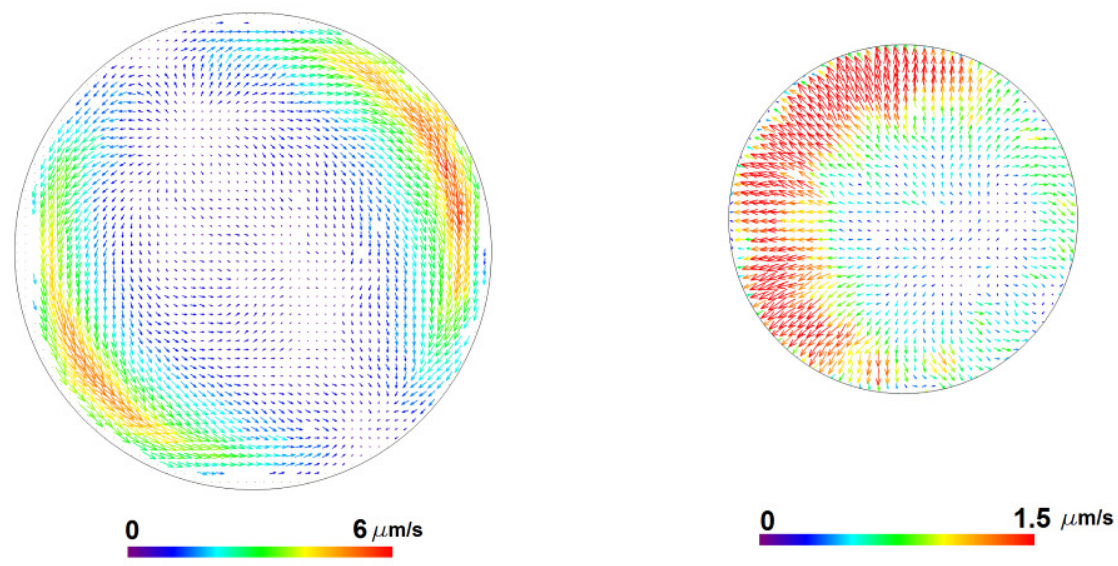

Figure 4. Instantaneous velocity fields in a droplet cross-section located at the distance $93 \mu \mathrm{m}$ from the substrate surface made of OTS. Left: $78 \mathrm{~s}$ after the beginning of evaporation. Right: $220 \mathrm{~s}$ after the beginning of evaporation. Full evaporation time was equal to $418 \mathrm{~s}$.

\section{References}

1. H. Erbil, Adv. Colloid Interface Sci., 170 (2012)

2. A.L. Karchevsky, I.V. Marchuk, O.A. Kabov, Appl. Math. Model., 40 (2), 1029-1037 (2016)

3. E.Ya. Gatapova., A.A. Semenov, D.V. Zaitsev, O.A. Kabov, Colloids and Surfaces A: Physicochemical and Engineering Aspects, 441, 776 (2014)

4. K. Hyoung Kang, S. Joon Lee, C. Mook Lee, I. Seok Kang, Meas. Sci. Technol., 15 (2004)

5. H. K. Dhavaleswarapu , C. P. Migliaccio, S. V. Garimella , J.Y. Murth y, Langmuir, 26(2) (2010)

6. P. J. Sáenz, K. Sefiane, J. Kim, O.K. Matar, P. Valluri, J.Fluid Mech., 772 (2015)

7. O.A. Kabov, D.V. Zaitsev, Doklady Physics, 58, 292 (2013) 